\title{
Polyp Prevention Trial
}

National Cancer Institute

\section{Source}

National Cancer Institute. Polyp Prevention Trial. NCI Thesaurus. Code C16163.

Investigated low fat, high fiber diets in preventing recurrence of colorectal polyps 